\title{
GK Persei and EX Hydrae: Intermediate polars with small magnetospheres
}

\author{
V. Suleimanov ${ }^{1}$, V. Doroshenko ${ }^{1}$, L. Ducci ${ }^{1,2}$, G.V. Zhukov ${ }^{3}$, and K. Werner ${ }^{1}$ \\ ${ }^{1}$ Institut für Astronomie und Astrophysik, Kepler Center for Astro and Particle Physics, Universität Tübingen, Sand 1, \\ 72076 Tübingen, Germany \\ e-mail: suleimanov@astro.uni-tuebingen.de \\ 2 ISDC Data Center for Astrophysics, Université de Genève, 16 chemin d'Écogia, 1290 Versoix, Switzerland \\ 3 Kazan (Volga region) Federal University, Kremlevskaja str., 18, 420008 Kazan, Russia
}

Received 12 February 2016 / Accepted 14 April 2016

\begin{abstract}
Observed hard X-ray spectra of intermediate polars are determined mainly by the accretion flow velocity at the white dwarf surface, which is normally close to the free-fall velocity. This allows us to estimate the white dwarf masses as the white dwarf mass-radius relation $M-R$ and the expected free-fall velocities at the surface are well known. This method is widely used. However, derived white dwarf masses $M$ can be systematically underestimated because the accretion flow is stopped at, and re-accelerates from, the magnetospheric boundary $R_{\mathrm{m}}$ and, therefore, its velocity at the surface is lower than free fall. To avoid this problem, we computed a two-parameter set of model hard X-ray spectra, which allows us to constrain a degenerate $M-R_{\mathrm{m}}$ dependence. Previous works showed that power spectra of accreting X-ray pulsars and intermediate polars exhibit breaks at frequencies corresponding to Keplerian frequencies at the magnetospheric boundary. Therefore, the break frequency $v_{\mathrm{b}}$ in an intermediate polar power spectrum gives another relation in the $M-R_{\mathrm{m}}$ plane. The intersection of the two dependences allows us, therefore, to determine the white dwarf mass and magnetospheric radius simultaneously. To verify the method, we analysed the archival Suzaku observation of EX Hya, obtaining $M / M_{\odot}=0.73 \pm 0.06$ and $R_{\mathrm{m}} / R=2.6 \pm 0.4$, which is consistent with the values determined by other authors. Subsequently, we applied the same method to a recent NuSTAR observation of another intermediate polar GK Per performed during an outburst and found $M / M_{\odot}=0.86 \pm 0.02$ and $R_{\mathrm{m}} / R=2.8 \pm 0.2$. The long duration observations of GK Per in quiescence performed by Swift $/$ BAT and INTEGRAL observatories indicate increase of magnetosphere radius $R_{\mathrm{m}}$ at lower accretion rates.
\end{abstract}

Key words. accretion, accretion disks - novae, cataclysmic variables - X-rays: binaries - methods: numerical

\section{Introduction}

Close binary systems consisting of a normal donor and a white dwarf (WD) accreting through Roche lobe overflow are called cataclysmic variables (CVs; see review in Warner 2003). Intermediate polars (IPs) are a subclass of CVs with moderately magnetized WDs $\left(B \sim 10^{4}-10^{6} \mathrm{G}\right)$. Central parts of accretion discs in these systems are destroyed by the WD magnetic field within its magnetosphere with radius $R_{\mathrm{m}}$. At smaller radii the accreted plasma couples to the field lines and forms a shock wave close to the WD magnetic poles. The shocked plasma is heated up to WD virial temperatures $(k T \sim 10-30 \mathrm{keV})$, cools through thermal bremsstrahlung, and settles down to the WD surface. As a result, IPs are bright hard X-ray sources (Revnivtsev et al. 2004a; Barlow et al. 2006; Landi et al. 2009). The hot post-shock region is optically thin and its averaged temperature can be estimated directly from the observed spectrum. The plasma temperature depends only on WD compactness and, therefore, provides a direct estimate of the WD mass (Rothschild et al. 1981).

A theory of the post-shock regions (PSR) on WDs was first considered by Aizu (1973) and further developed in several works where the WD gravity, influence of cyclotron cooling, dipole geometry of the magnetic field, and difference between the temperatures of the electron-ion plasma components were taken into account (Fabian et al. 1976; Wu et al. 1994; Cropper et al. 1999; Canalle et al. 2005; Saxton et al. 2007; Hayashi \& Ishida 2014a). Some of these models were used to estimate the WD masses in several intermediate polars and polars (Cropper et al. 1998, 1999; Ramsay 2000; Revnivtsev et al. 2004b; Suleimanov et al. 2005; Falanga et al. 2005; Brunschweiger et al. 2009; Yuasa et al. 2010; Hayashi \& Ishida 2014b). However, in all these models the accreting plasma was assumed to fall from infinity whereas in reality the magnetospheric radius could be small enough (a few WD radii) to break this assumption. As a consequence the accretion flow is accelerated to lower velocities and the postshock region has lower temperatures for the given WD parameters. Therefore, WD masses derived from previous PSR models can be underestimated.

Such possibility was first suggested by Suleimanov et al. (2005) for GK Per to explain a significant discrepancy between the WD mass estimated using Rossi X-ray Timing Explorer (RXTE) observations of an outburst and optical spectroscopy. Later Brunschweiger et al. (2009) confirmed this conclusion by estimating the WD mass in GK Per in quiescence. The difference between the estimates of the WD mass in EX Hya obtained using the RXTE observations $\left(0.5 M_{\odot}\right.$, Suleimanov et al. 2005) and from the optical observations $\left(0.79 M_{\odot}\right.$, Beuermann \& Reinsch 2008) was also attributed to a small magnetospheric radius in this system ( 2.7 WD radii; see e.g., Revnivtsev et al. 2011).

Here we present a new set of IP model spectra that, for the first time, quantitatively account for the finite size of the magnetosphere. The models are calculated for a set of magnetospheric 
radii (expressed in units of WD radii) assuming a relatively high local mass accretion rate $\left(>1 \mathrm{~g} \mathrm{~s}^{-1} \mathrm{~cm}^{-2}\right)$. Additional cyclotron cooling was ignored as it is not important in such conditions. The model is publicly available and allows direct investigations of the dependence of magnetospheric radius on mass accretion rate in IPs.

A new method of simultaneous determination of the WD mass and the magnetospheric radius is suggested on the basis of this set. We propose to add information about the observed frequency of a break in the power spectra of IP X-ray light curves. Using spectral fitting and this additional information we can constrain the relative magnetospheric radius together with the WD mass. We verified the method using high quality Suzaku observations of the well-studied IP EX Hya and, subsequently applied it to study the spectra of the IP GK Per in outburst and quiescence.

\section{The method}

The basic commonly accepted physical picture of the X-ray emitting region in intermediate polars is that matter falls along magnetic field lines onto the WD and forms an adiabatic shock above the surface. The free-fall velocity of the matter $v_{\mathrm{ff}}$ decreases by a factor of four as it crosses the shock according to Rankine-Hugoniot relations. The rest kinetic energy transforms to internal gas energy and heats the matter up to the temperature

$k T_{0}=\frac{3}{16} \mu m_{\mathrm{H}} v_{\mathrm{ff}}^{2}=\frac{3}{8} \mu m_{\mathrm{H}} \frac{G M}{R}\left(1-r_{\mathrm{m}}{ }^{-1}\right)$.

Here $M$ and $R$ are the mass and radius of the WD, $r_{\mathrm{m}}=R_{\mathrm{m}} / R$ is the relative radius of the magnetosphere, $\mu=0.607$ is the mean molecular weight for a completely ionized plasma with solar chemical composition, and $m_{\mathrm{H}}$ and $k$ are the proton mass and the Boltzmann constant. Here we take into account that the matter starts falling from the finite distance $R_{\mathrm{m}}$ from the WD. The heated matter settles down to the WD surface in the subsonic regime and loses energy by optically thin bremsstrahlung. The cooling due to cyclotron emission is insignificant in intermediate polars owing to a relatively weak WD magnetic field and can be ignored. The height of the shock wave above the WD surface is determined by the cooling rate and models of the post-shock region can be accurately computed together with emergent spectra (see next section).

The observed hard X-ray spectra of intermediate polars can be well approximated with thermal bremsstrahlung. The temperature of the bremsstrahlung $k T_{\text {br }}$ is, however, lower in comparison with $k T_{0}$ and a proportional factor $A$ between these temperatures can be found from accurate PSR computations alone. The computations and their comparison with the observed X-ray spectra presented later give

$k T_{\mathrm{br}}=A k T_{0} \approx 0.64 k T_{0}$.

The WD radius depends on the WD mass, see e.g. Nauenberg (1972),

$R=7.8 \times 10^{8} \mathrm{~cm}\left(\left(\frac{1.44}{m}\right)^{2 / 3}-\left(\frac{m}{1.44}\right)^{2 / 3}\right)^{1 / 2}$,

where $m=M / M_{\odot}$ is the WD mass in units of solar masses. Therefore, Eqs. (1)-(3) define a curve in the $m-r_{\mathrm{m}}$ plane, which corresponds to the observed bremsstrahlung temperature



Fig. 1. Curves of constant bremsstrahlung temperature (6) and constant break frequency (7) on the $m-r_{\mathrm{m}}$ plane. Intersection of the curves provides an estimate of the WD mass and the magnetospheric radius.

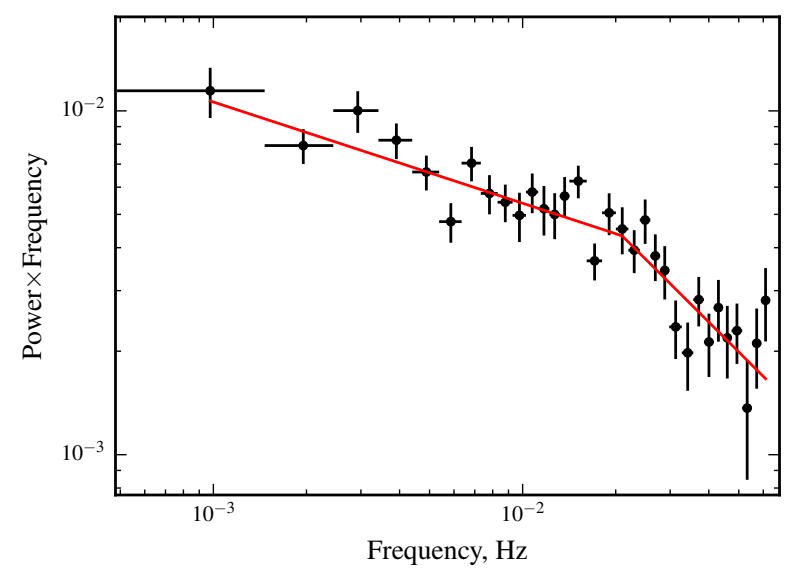

Fig. 2. Power spectrum of the $0.5-10 \mathrm{keV} X$-ray light curve of EX Hya observed by Suzaku. The break frequency $v_{\mathrm{b}}=0.021 \pm 0.006$ is consistent with the value reported by Revnivtsev et al. (2011) using RXTE data.

$k T_{\text {br }}$ (see. Fig. 1). Another curve on the $m-r_{\mathrm{m}}$ plane can be obtained from X-ray light curve analysis. Revnivtsev et al. (2009, 2011) showed that power spectra of X-ray pulsars and intermediate polars exhibit a break at the frequency $v_{\mathrm{b}}$ corresponding to the Kepler frequency at $R_{\mathrm{m}}$,

$v_{\mathrm{b}}^{2}=\frac{G M}{4 \pi^{2} R_{\mathrm{m}}^{3}}$.

An example of the power spectrum with the break obtained for the intermediate polar EX Hya using the observations performed by Suzaku observatory (see Sect. 6.1) is presented in Fig. 2. The curve described by Eq. (4) is also shown in Fig. 1. The intersection of the two curves allows us to estimate the WD mass and the magnetospheric radius of the investigated intermediate polar.

Equations (1)-(3) and (4) can be simplified using the linear approximation of the WD mass-radius relation presented by Suleimanov et al. (2008)

$R \approx 1.364 \times 10^{9} \mathrm{~cm}(1-0.59 \mathrm{~m})$,

which is valid for WD masses in the $0.4-1.2 M_{\odot}$ range. The resulting relations are

$m \approx \frac{k T_{\mathrm{br}}}{15\left(1-r_{\mathrm{m}}^{-1}\right)+0.59 k T_{\mathrm{br}}}$, 
where $k T_{\text {br }}$ is measured in $\mathrm{keV}$, and

$r_{\mathrm{m}} \approx 1.5\left(\frac{v_{\mathrm{b}}}{0.02}\right)^{-2 / 3} \frac{m^{1 / 3}}{1-0.59 m}$

The accuracy of these relations can be checked using available data. The hard X-ray spectrum of the intermediate polar V1223 Sgr was fitted by a bremsstrahlung spectrum with $k T_{\text {br }} \approx 29 \mathrm{keV}$ (Revnivtsev et al. 2004a). We get $m \approx 0.9$ using relation (6) with $r_{\mathrm{m}}=\infty$, whereas the direct fitting with PSR spectra gave $0.95 \pm 0.05$ (Suleimanov et al. 2005). Revnivtsev et al. (2011) found $r_{\mathrm{m}} \approx 2.7$ for EX Hya using fixed $m=0.79$ and $v_{\mathrm{b}}=0.02$. The relation (7) gives $r_{\mathrm{m}} \approx 2.6$.

Thus, relations (6) and (7) allow to evaluate the WD mass and the magnetospheric radius using a simple X-ray spectrum fitted by bremsstrahlung and break frequency in the power spectrum. However, direct fitting of PSR model spectra is necessary for more accurate results. Of course, we use the accurate $M-R$ relation further on and provide the relations above for convenience.

\section{The model of the post-shock region}

\subsection{Basic equations}

The post-shock region can be fully described using the following set of equations (see, for example, Mihalas 1978). The continuity equation is

$\boldsymbol{\nabla} \cdot(\rho \boldsymbol{v})=0$,

where $\rho$ is the plasma density and $\boldsymbol{v}$ is the vector of the gas velocity. Conservation of momentum for each gas element is described by the vector Euler equation

$\rho \boldsymbol{v} \cdot \boldsymbol{\nabla v}=-\boldsymbol{\nabla} P+\boldsymbol{f}$,

where $P$ is the gas pressure (we ignore the radiation pressure here) and $f$ is the force density. The energy equation for the gas is

$\boldsymbol{\nabla} \cdot\left[\left(\frac{1}{2} \rho v^{2}+\varepsilon+P\right) \boldsymbol{v}\right]=\boldsymbol{f} \cdot \boldsymbol{v}-\boldsymbol{\nabla} \cdot \boldsymbol{q}$.

Here $\varepsilon=(3 / 2) P$ is the density of internal gas energy. The first term on the right side of the energy equation is the power density and the second term accounts for radiative energy loss ( $\boldsymbol{q}$ is the vector of the radiation flux). These equations must be supplemented by the ideal-gas law

$P=n k T=\frac{\rho k T}{\mu m_{\mathrm{H}}}$,

where $n$ is the total number density of particles.

Here we consider the one-dimension optically thin flow in a white dwarf gravitational field. Therefore, we substitute the radiation loss term by the local radiative cooling function $\Lambda(\rho, T)$

$\boldsymbol{\nabla} \cdot \boldsymbol{q}=\Lambda(\rho, T)=n_{\mathrm{i}} n_{\mathrm{e}} \Lambda_{\mathrm{N}}(T)$,

where

$n_{\mathrm{e}}=\frac{\rho}{\mu_{\mathrm{e}} m_{\mathrm{H}}}$

is the electron number density, and

$n_{\mathrm{i}}=n-n_{\mathrm{e}}=\frac{\rho}{m_{\mathrm{H}}}\left(\frac{1}{\mu}-\frac{1}{\mu_{\mathrm{e}}}\right)$ is the ion number density. Here $\mu_{\mathrm{e}}=1.167$ is the mean number of nucleons per electron for fully ionized solar mix plasma. The universal cooling function was computed by many authors and in previous work (Suleimanov et al. 2005) we used $\Lambda_{\mathrm{N}}$ computed by Sutherland \& Dopita (1993). In the present work, we use a more modern cooling function computed by the code APEC (Smith et al. 2001) using the database $A$ tomDB ${ }^{1}$ for a solar chemical composition. In the previous work (Suleimanov et al. 2005), the total cooling function was overestimated by factor $\approx 4$, as was correctly mentioned by Hayashi \& Ishida (2014a) because $n^{2}$ instead of $n_{\mathrm{i}} n_{\mathrm{e}}$ was used in Eq. (12). Fortunately, this error did not influence the emergent model spectra and the obtained WD masses (see Appendix). It was corrected in subsequent work (Suleimanov et al. 2008).

We assume that only gravity force operates in the PSR written as

$f=-\frac{G M}{(R+z)^{2}} \rho=-g(z) \rho$.

Here $z$ is geometrical height above the WD surface and the considered accretion flow settles along $z$.

\subsection{Quasi-dipole geometry}

We use the approximation of the dipole geometry suggested by Hayashi \& Ishida (2014a). They assumed that the PSR crosssection $S$ depends on $z$ as follows: $S \sim z^{n}$. In this case every divergence in hydrodynamical equations can be written as $\boldsymbol{\nabla} \cdot y=S^{-1} \mathrm{~d}(S y) / \mathrm{d} z$,

$\frac{1}{S} \frac{\mathrm{d}(S \rho \mathrm{v})}{\mathrm{d} z}=0$

$\rho v \frac{\mathrm{d} v}{\mathrm{~d} z}=-\frac{\mathrm{d} P}{\mathrm{~d} z}-g(z) \rho$,

and

$\frac{1}{S} \frac{\mathrm{d}}{\mathrm{d} z}\left[S v\left(\frac{1}{2} \rho v^{2}+\varepsilon+P\right)\right]=-g(z) \rho v-\Lambda(\rho, T)$.

If we take $S \sim z^{2}$, we obtain the well-known equations for the spherically symmetric geometry. The dependence $S \sim z^{3}$ mimics the dipole geometry.

Equation (16) has the integral

$S \rho v=a$,

where $a$ is the local mass accretion rate at the WD surface, $[a]=\mathrm{g} \mathrm{s}^{-1} \mathrm{~cm}^{-2}$. Using this integral, we can replace $\rho$ in the next two equations by $a / S v$ and finally we have

$v \frac{\mathrm{d} v}{\mathrm{~d} z}+\frac{S v}{a} \frac{\mathrm{d} P}{\mathrm{~d} z}=-g(z)$,

and

$v \frac{\mathrm{d} P}{\mathrm{~d} z}+\gamma P \frac{\mathrm{d} v}{\mathrm{~d} z}+\gamma \frac{P v}{S} \frac{\mathrm{d} S}{\mathrm{~d} z}=-(\gamma-1) \Lambda(\rho, T)$,

where $\gamma=5 / 3$ is the adiabatic index. We note that Eq. (20) coincides with the corresponding equation in Hayashi \& Ishida (2014a) with the exception of the energy conservation law. However, our Eq. (21) coincides with the energy equation in Canalle et al. (2005) written for the PSR at the magnetic pole (in

1 http://www . atomdb.org 
this case $\frac{1}{h_{2}} \frac{\mathrm{d}}{\mathrm{d} \omega}=\frac{\mathrm{d}}{\mathrm{d} z}$ ), if we take into account that their product $h_{1} h_{3}$ corresponds to our function $S$.

Equations (20) and (21) can be rewritten as follows:

$\frac{\mathrm{d} v}{\mathrm{~d} z}=\frac{(\gamma-1) S \Lambda(\rho, T)+a g(z)-\gamma P v \mathrm{~d} S / \mathrm{d} z}{S \gamma P-a v}$

and

$\frac{\mathrm{d} P}{\mathrm{~d} z}=-g(z) \frac{a}{S v}-\frac{a}{S} \frac{\mathrm{d} v}{\mathrm{~d} z}$,

where

$S=\left(\frac{R+z}{R}\right)^{3}$

and

$\frac{\mathrm{d} S}{\mathrm{~d} z}=3 \frac{(R+z)^{2}}{R^{3}}$.

\subsection{Method of solution}

Equations (22) and (23) can be solved with appropriate boundary conditions. We use a commonly accepted suggestion about a strong adiabatic shock. In particular, at the upper PSR boundary $\left(z=z_{0}\right)$ we have the following boundary conditions

$v_{0}=-\frac{1}{4} v_{\mathrm{ff}}\left(z_{0}\right), \quad P_{0}=-3 \frac{a v_{0}}{S\left(z_{0}\right)}, \quad T_{0}=3 \frac{\mu m_{\mathrm{H}}}{k} v_{0}^{2}$

and

$v=0$

at the WD surface $(z=0)$. The free-fall velocity at the upper PSR boundary $v_{\mathrm{ff}}\left(z_{0}\right)$ depends on the WD compactness and the inner disc radius, or the magnetosphere radius $R_{\mathrm{m}}$

$\mathrm{v}_{\mathrm{ff}}\left(z_{0}\right)=\sqrt{2 G M}\left(\frac{1}{R+z_{0}}-\frac{1}{R_{\mathrm{m}}}\right)^{1 / 2}$.

Therefore, the input parameters for each PSR model are the WD mass $M$, the local mass accretion rate at the WD surface $a$, and the magnetospheric radius $R_{\mathrm{m}}$. We reduced the number of parameters of the model by eliminating the WD radius using the WD mass-radius relation of Nauenberg (1972).

We computed each PSR model using a logarithmically equidistant grid over $z$. We start from the first point with very small $z_{1}=0.1 \mathrm{~cm}$. We fix the temperature $T_{1}=$ $300000 \mathrm{~K}$ and some pressure $P_{1}$ at this point. The corresponding density $\rho_{1}$ and velocity $v_{1}$ are found using the mass conservation law (19) and the equation of the state (11). Then Eqs. (22) and (23) are integrated up to the height $z$, where $v(z)=0.25 v_{\mathrm{ff}}(z)$. At that point the pressure also has to be equal to $-3 a v(z) / S(z)$. However, the last condition does not hold for the arbitrarily chosen $P_{1}$. Therefore, we find the required pressure at the first point $P_{1}$ in the range $0.1-30 P_{0}$ by the dichotomy method. Once both aforementioned upper boundary conditions are satisfied with a relative accuracy better than $10^{-8}$, we take the height $z$ as the PSR height $z_{0}$. The results depend slightly on the choice of the starting point (see Fig. 3). In the previous paper (Suleimanov et al. 2005), models were computed starting from the highest PSR point $z_{0}$. Both methods give coincident solutions at $k T>0.5 \mathrm{keV}$, but the new method describes the bottom part of the PSR much better.

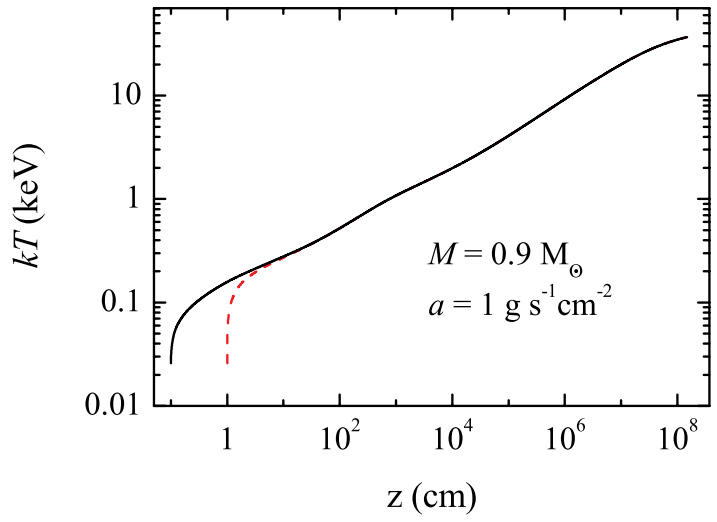

Fig. 3. Comparison of the temperature structures of the models computed for two different values of the first model point, $z_{1}=0.1 \mathrm{~cm}$, solid curve, and $z_{1}=1 \mathrm{~cm}$, dashed curve.

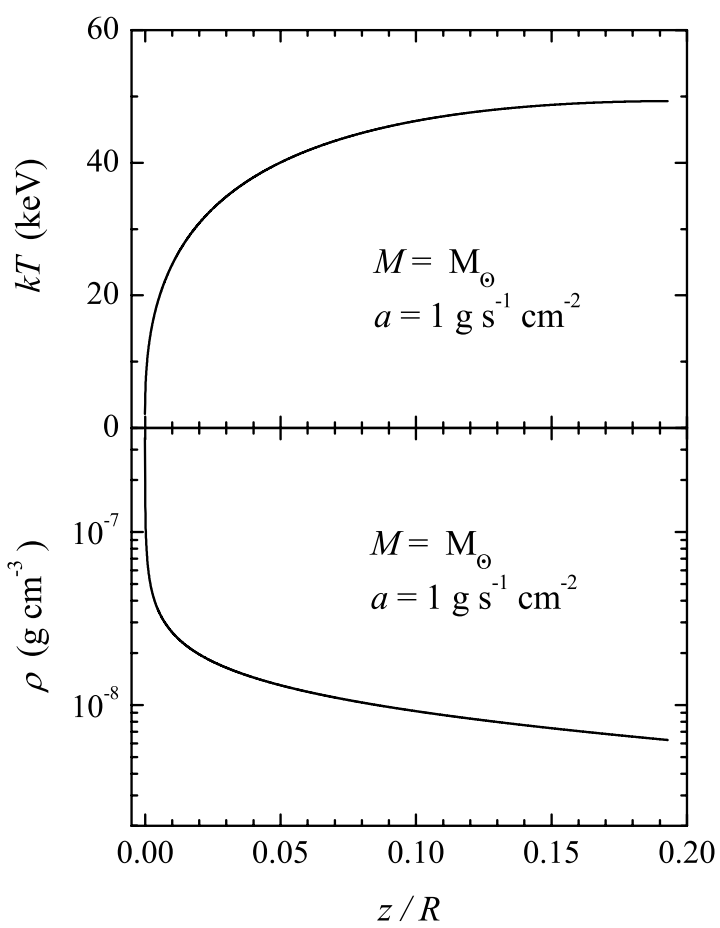

Fig. 4. Distributions of temperature and density in the model with $M=$ $M_{\odot}$ and local mass accretion rate $a=1 \mathrm{~g} \mathrm{~s}^{-1} \mathrm{~cm}^{-2}$. See similar Fig. 2 in Hayashi \& Ishida (2014a).

The code allows us to compute models in the cylindrical geometry as well. The computation of a such model with the same parameters as used by Hayashi \& Ishida (2014a) for one of their models gives similar results (compare Figs. 4 with 2 in Hayashi \& Ishida 2014a). We also find a good agreement between our results and those obtained by Canalle et al. (2005) for the dipole geometry (compare Figs. 5 with 6 in Canalle et al. 2005).

However, the results obtained by Hayashi \& Ishida (2014a) for the dipole geometry are physically incorrect. They found that the PSR heights are lower in the dipole geometry. However, in the dipole geometry scenario, the plasma cools slowly because of its lower density compared to the cylindrical geometry case. Therefore, the PSR height in the dipole geometry is expected to be higher. 

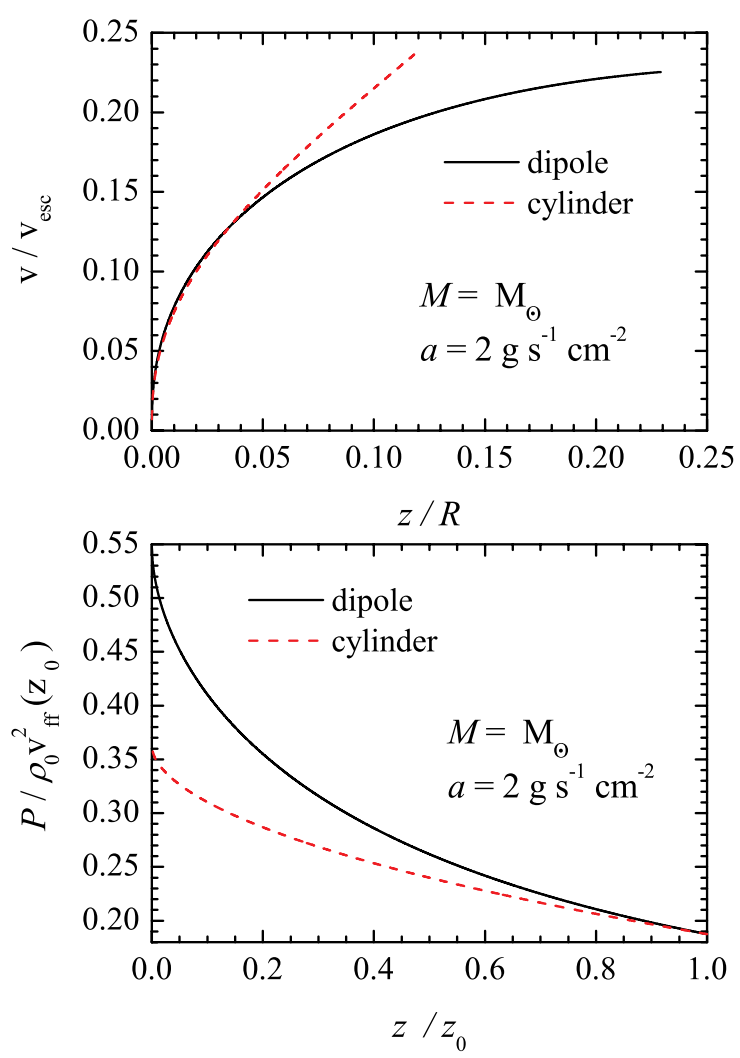

Fig. 5. Comparison of the models computed in cylindrical and dipole geometries. This result reproduces Figs. 6 and 4 in Canalle et al. (2005). Here $v_{\text {esc }}=\sqrt{2 G M / R}$.

\subsection{Spectra computations}

We computed the PSR model spectra assuming solar abundances and fully ionized of all the abundant elements in the PSR. This assumption is reasonable at temperatures $k T>1 \mathrm{keV}$, therefore, the computed spectra are correct at relatively high energies only $(E>3 \mathrm{keV})$. At lower energies, the spectra are dominated by numerous emission spectral lines and photo-recombination continua (see e.g. Canalle et al. 2005). The PSR models are optically thin and the relative spectra can be calculated with a simple integration of the local (at the given height $z$ ) emissivity coefficients $\eta_{\mathrm{E}}=k_{\mathrm{E}} B_{\mathrm{E}}$ and taking into account the dipole geometry

$F_{\mathrm{E}}=\int_{0}^{z_{0}} k_{\mathrm{E}} B_{\mathrm{E}} S \mathrm{~d} z$

where $B_{\mathrm{E}}$ is Planck function. The free-free opacities $k_{\mathrm{E}}$ for the 15 most abundant chemical elements were computed using Kurucz's code ATLAS (Kurucz 1970, 1993) modified for high temperatures (see details in Ibragimov et al. 2003; Suleimanov \& Werner 2007).

Examples of model spectra are presented in Figs. 6 and 7. The first model shows that spectra have a degeneracy with respect to the local mass accretion rate and are almost indistinguishable for any $a>1 \mathrm{~g} \mathrm{~s}^{-1} \mathrm{~cm}^{-2}$. The model spectrum computed for $a=0.2 \mathrm{~g} \mathrm{~s}^{-1} \mathrm{~cm}^{-2}$ is, however, slightly softer. Therefore, we can compute the model spectra for every WD mass with one sufficiently high $a$ and it would be sufficient to evaluate the WD mass by fitting its hard X-ray spectrum with the computed PSR model spectra. In contrast, different inner disc or magnetospheric radii are very important and can influence the WD mass evaluation significantly (Fig. 7).

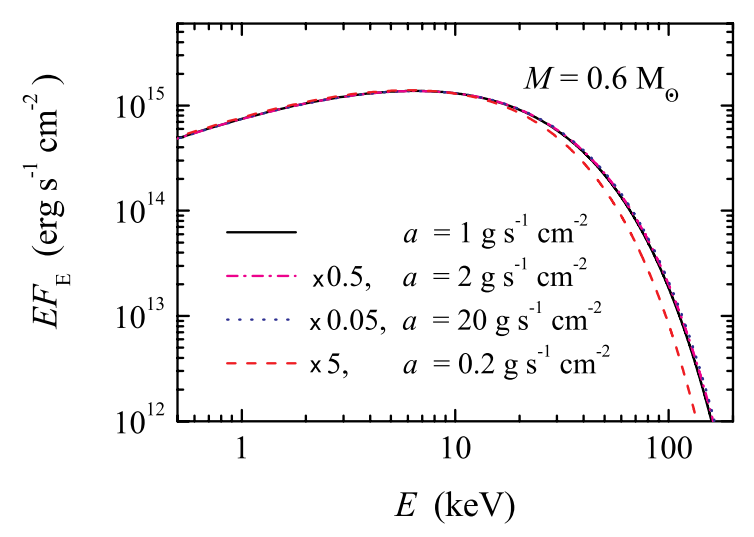

Fig. 6. Comparison of model spectra for several local mass accretion rates and a fixed WD mass. Spectra are normalized to the spectrum with $a=1 \mathrm{~g} \mathrm{~s}^{-1} \mathrm{~cm}^{-2}$.

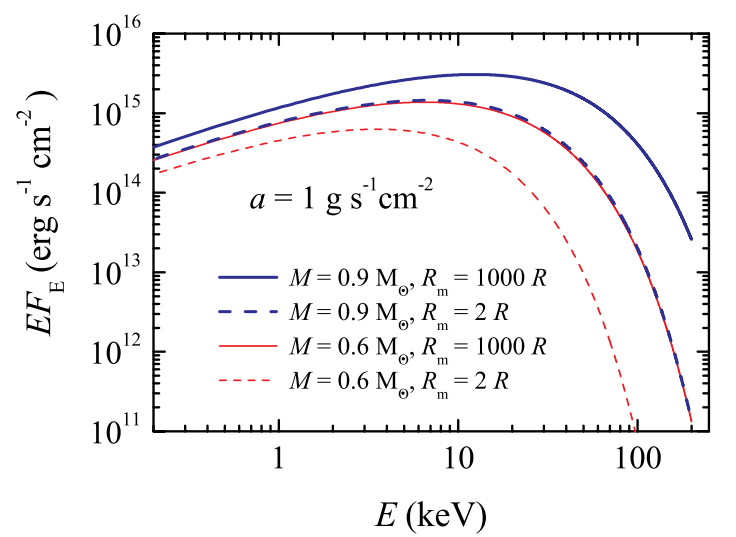

Fig. 7. Comparison of model spectra computed for two WD masses, 0.6 (thin curves) and 0.9 (thick curves) $M_{\odot}$ and two relative magnetospheric radii, $R_{\mathrm{m}} / R=2$ (dashed curves) and 1000 (solid curves). The local mass accretion rate is fixed to $a=1 \mathrm{~g} \mathrm{~s}^{-1} \mathrm{~cm}^{-2}$ for all the computed spectral models.

Using the described method we computed a set of PSR model spectra for a grid of two input parameters, the WD mass $M$, and the relative magnetospheric radius $R_{\mathrm{m}} / R$. The maximum PSR temperature $T_{0}$ strongly depends on the factor $1-R / R_{\mathrm{m}}$; see Eqs. (26) and (28). Therefore, the equidistant $T_{0}$ subgrid for a given WD mass has to be proportional to $R_{\mathrm{m}} / R \sim N_{\max } / N$. We chose the subgrid with $N_{\max }=60$ and $N$ changing from 40 (which corresponds to $R_{\mathrm{m}} / R=1.5$ ) to 1 $\left(R_{\mathrm{m}} / R=60\right)$. The additional model with $R_{\mathrm{m}} / R=1000$ was included to represent the pseudo-infinity magnetospheric radius. The grid was computed for 56 values of WD mass from 0.3 to $1.4 M_{\odot}$ with a step of $0.02 M_{\odot}$, i.e. 2296 models in total. Every PSR model was computed for a fixed mass accretion rate $\dot{M}=10^{16} \mathrm{~g} \mathrm{~s}^{-1}$ and fixed ratio of the PSR footprint area to the WD surface $f=S_{\mathrm{PSR}} / 4 \pi R^{2}=5 \times 10^{-4}$. The local mass accretion rate changes from $a \approx 1 \mathrm{~g} \mathrm{~s}^{-1} \mathrm{~cm}^{-2}$ for the lightest WD to $a \approx 70 \mathrm{~g} \mathrm{~s}^{-1} \mathrm{~cm}^{-2}$ for the heaviest WD in accordance with decreasing WD radius. This model grid can be used to estimate the WD mass at a fixed $R_{\mathrm{m}} / R$ by fitting an IP hard X-ray spectrum. In addition, the fit returns the following normalization of the spectrum:

$K=\frac{f R^{2}}{d^{2}}$ 
The grid will be distributed as an XSPEC additive table model ${ }^{2}$ and publicly available to the scientific community.

\section{Investigated objects}

\subsection{EX Hya}

This intermediate polar is one of the closest CVs with a distance of $d \approx 65 \mathrm{pc}$ and a low accretion luminosity $L_{\mathrm{acc}} \approx$ $2.6 \times 10^{32} \mathrm{erg} \mathrm{s}^{-1}$ (Beuermann et al. 2003). The orbital period of EX Hya is relatively short $\left(P_{\text {orb }}=98.26 \mathrm{~min}\right)$, whereas the spin period is relatively long $\left(P_{\mathrm{s}}=67.03 \mathrm{~min}\right)$ in comparison with other intermediate polars. The WD mass in this object is well determined from optical observations $\left(M / M_{\odot}=0.79 \pm\right.$ 0.03; Beuermann \& Reinsch 2008). Recently, Revnivtsev et al. (2011) found that the magnetospheric radius in EX Hya is small, $R_{\mathrm{m}} / R \approx 2.7$. A similar value $\left(R_{\mathrm{m}} / R \approx 2.5\right)$ was found before by Belle et al. (2003). Moreover, Siegel et al. (1989) found the centre of the optical radiation in this system is situated at a distance of about $1.5 \times 10^{9} \mathrm{~cm}$ from the WD centre. Most probably, this light arises as a result of irradiation of the inner disc rim with the hard X-ray emission of the PSR. This distance again corresponds to $\sim 2 R$ for the $0.8 M_{\odot}$ white dwarf. Permanent increasing of the white dwarf spin period provides other evidence of the small magnetospheric radius (Hellier \& Sproats 1992). This is possible if the magnetospheric radius is much smaller than the corotation radius, which is indeed very large for EX Hya $(\sim 50 R)$. If the corotation radius is close to the magnetospheric radius then the intermediate polar will be close to the equilibrium with changing spin-up and spin-down periods (see e.g. the case of FO Aqr in Patterson et al. 1998).

Such a small magnetospheric radius can explain the small WD masses obtained from the fit of the observed spectra with PSR models $\left(0.5 \pm 0.05 M_{\odot}\right.$; Suleimanov et al. 2005 ; and $0.42 \pm$ 0.02 , Yuasa et al. 2010) when an infinity magnetospheric radius was used in the computations. The low absorbed X-ray spectrum of EX Hya enables detailed investigation of the spectral lines (see e.g. Luna et al. 2015). These properties make EX Hya an ideal target for testing the method of simultaneous determination of the WD mass and the magnetospheric radius proposed in this paper.

\subsection{GK Per}

The intermediate polar GK Per (Watson et al. 1985) has a very long orbital period $\left(P_{\text {orb }} \approx 1.99\right.$ days $)$ and a $\mathrm{K} 1$ subgiant secondary star (Warner 1976; Crampton et al. 1986; Morales-Rueda et al. 2002). GK Per is also classified as a dwarf nova with very long outbursts $(\approx 50$ days) repeated roughly every three years (Šimon 2002). GK Per is also known as Nova Persei 1901 and based on ejecta studies, Warner (1976) determined a distance to GK Per of 460 pc that is consistent with a previous determination (470 pc) made by McLaughlin (1960).

The spin period of the WD in GK Per is relatively short $\left(P_{\mathrm{s}} \approx 351 \mathrm{~s}\right.$; Watson et al. 1985; Mauche 2004). The pulse profile shape changed from a single-peaked during the outbursts (Hellier et al. 2004) to a two-peaked type in quiescence (Patterson 1991; Ishida et al. 1992). The most likely explanation for these variations is the obscuration of the second WD pole by a dense accretion curtain during the outbursts

\footnotetext{
2 https://heasarc.gsfc.nasa.gov/xanadu/xspec/models/ ipolar.htmll
}

(Hellier et al. 2004; Vrielmann et al. 2005). During the outbursts, quasi-periodic flux oscillations with a typical timescale $\sim 5000 \mathrm{~s}$ were reported both in X-rays (Watson et al. 1985) and in the emission line spectrum in the optical band (Morales-Rueda et al. 1999).

Analysis of the absorption line spectra of the GK Per secondary during quiescence allowed to determine the stellar mass ratio in the system $(q=0.55 \pm 0.21)$ and set lower limits for the binary components, $M / M_{\odot} \geq 0.84 \pm 0.24$ and $M_{\mathrm{sec}} / M_{\odot} \geq$ $0.48 \pm 0.32$ (Morales-Rueda et al. 2002). These values are in accordance with the masses obtained by Crampton et al. (1986), $M / M_{\odot} \approx 0.9 \pm 0.2$ and $M_{\mathrm{sec}} / M_{\odot} \approx 0.25$. A similar WD mass estimate was obtained by Reinsch (1994), $M / M_{\odot} \geq 0.78$. The large uncertainties in mass determination are mostly due to the poorly constrained inclination of the orbital plane of the system to line of sight $i$, which was evaluated to be between $73^{\circ}$ and $\sim 50^{\circ}$ (Reinsch 1994; Morales-Rueda et al. 2002). Warner (1986) claimed $i \sim 75^{\circ}$ using the correlation between the equivalent widths of some emission lines and the inclination angle. This high inclination angle is preferable from our point of view because the optical emission lines $\left(\mathrm{H}_{\beta}\right.$, HeII 4686, and others) are also observed in emission during the outbursts and no wide absorption wings are observed (Crampton et al. 1986; Morales-Rueda et al. 1999). This behaviour is typical for highinclined CVs with high mass accretion rates (e.g. UX UMa; Neustroev et al. 2011). Therefore, the WD mass is likely close to the lower limit in the allowed region, $M / M_{\odot} \approx 0.9$.

It is also possible to determine the WD masses in old novae by comparing the observed optical light curves of nova outbursts with those predicted by theoretical models. Using this approach Hachisu \& Kato (2007) determined for GK Per a WD mass of $M / M_{\odot}=1.15 \pm 0.05$. However, this result depends on the assumed hydrogen mass fraction $(X=0.54)$ in the expanded Nova Persei 1901 envelope.

GK Per is a bright hard X-ray source during the outbursts and it was observed by many X-ray observatories including EXOSAT (Watson et al. 1985; Norton et al. 1988), Ginga (Ishida et al. 1992), ASCA (Ezuka \& Ishida 1999), RXTE (Hellier et al. 2004; Suleimanov et al. 2005), XMM-Newton (Vrielmann et al. 2005; Evans \& Hellier 2007), Chandra (Mauche 2004), INTEGRAL (Barlow et al. 2006; Landi et al. 2009), and Swift/BAT (Brunschweiger et al. 2009). Some of these observations were also used to estimate the WD mass.

The first measurements of the WD mass from X-ray data were based on the cylindrical PSR models (Suleimanov et al. 2005) and iron line diagnostic method (Ezuka \& Ishida 1999). The estimated WD masses turned out to be relatively low $\left(0.59 \pm 0.05\right.$ and $0.52 M_{\odot}$, respectively) and inconsistent with previous estimates based on optical observations ( 0.9 $M_{\odot}$; Crampton et al. 1986; Morales-Rueda et al. 2002). Suleimanov et al. (2005) attributed this discrepancy to the fact that the PSR models used to estimate the mass were computed assuming an accretion flow falling from infinity $\left(R_{\mathrm{m}}=\infty\right.$ in Eq. (28)), while in reality the corotation radius in this system should be smaller than $\sim 10 R$. Moreover, the magnetospheric radius has to be even smaller during the outburst, as the observed $\mathrm{X}$-ray flux increases by more than a magnitude with respect to quiescence (Ishida et al. 1992). Therefore, the accreting matter is expected to have lower kinetic energy at the WD surface and reach lower temperatures after the shock, so the WD mass is underestimated if one assumes that the accretion flow accelerates from infinity. 
Brunschweiger et al. (2009) used Swift/BAT observations during the low-flux state of GK Per and took the reduction of the kinetic energy into account. They obtained an improved WD mass value $0.90 \pm 0.12 M_{\odot}$, which isconsistent with estimates based on optical spectroscopy. Nevertheless, recent Suzaku observations of GK Per before and during the latest outburst do not show any significant differences between the spectrum in quiescence just before the outburst and the spectrum on the middle of the flux rise (Yuasa et al. 2016). This behaviour of GK Per has to be investigated further.

In the next sections we revisit this estimate and investigate the dependence of the magnetospheric radius on X-ray luminosity using the archival RXTE and Swift/BAT observations and the self-consistent PSR models presented in Sect. 3.

\section{Observations and data analysis}

\subsection{Suzaku observations of EX Hya}

Suzaku is equipped with the four-module X-ray Imaging Spectrometer (XIS; Koyama et al. 2007) covering the $0.2-12 \mathrm{keV}$ energy range, and a collimated Hard X-ray Detector (HXD) covering the $10-70 \mathrm{keV}$ and 50-600 keV energy range with PIN and GSO detectors (Kokubun et al. 2007; Takahashi et al. 2007). Suzaku observed EX Hya on 8 July 2007 for $\sim 100 \mathrm{ks}$ (obsid \#402001010) with effective exposure of $\sim 75 \mathrm{ks}$ for XIS and $85 \mathrm{ks}$ for HXD PIN. The source is not detected significantly in the GSO energy band, so we restricted the analysis to XIS and HXD PIN data.

We follow standard procedures for the data reduction and employ default filtering criteria as described in the Suzaku data reduction guide ${ }^{3}$. We use the HEASOFT 6.16 software package with the current instrument calibration files (CALDB version 20151105). For HXD PIN background subtraction we adopted the "tuned" non-X-ray background (NXB) event file provided by the HXD team. We ignored the contribution of cosmic X-ray background $(\mathrm{CXB})$ as it is negligible compared to the source count rate.

\subsection{Observations of GK Per}

To investigate the properties of the source in outburst we use the recent $\sim 80 \mathrm{ks}$ long NuSTAR target of opportunity observation performed in April 2015 (obsid 90001008002 , PI is Marina Orio, some results of the observation are presented by Zemko et al. 2015, 2016). We use the HEASOFT 6.16 software package and current instrument calibration files (CALDB version 20151105). We follow the standard procedures described in the instruments data analysis software guide ${ }^{4}$ to screen the data and extract light curves and spectra. Total useful exposure after screening is $\sim 42 \mathrm{ks}$. We combine the data from the two NuSTAR units for timing analysis, whereas we extract and model spectra from the two units separately for spectral analysis.

We note that another observation was performed with NuSTAR in quiescence (Sept. 2015, obsid 30101021002), however, the data are not public. Therefore, to assess the source properties outside of the outburst we rely on Swift/BAT (Barthelmy et al. 2005) and INTEGRAL (Winkler et al. 2003) data. The Swift/BAT 70-month survey (Baumgartner et al. 2013)

\footnotetext{
https://heasarc.gsfc.nasa.gov/docs/suzaku/analysis/ abc/

4 http://heasarc.gsfc.nasa.gov/docs/nustar/analysis/ nustar_swguide.pdf
}

provides mission-long spectra and light curves for all detected sources including GK Per (listed as SWIFT J0331.1+4355). The total effective exposure for GK Per is $9.5 \mathrm{Ms}$. The analysis of the AAVSO light curve of the source shows that three outbursts occurred during the lifetime of the mission and the averaged spectrum includes these intervals. On the other hand, total duration of outbursts $(1.4 \mathrm{Ms})$ and average flux are comparatively low at $\sim 4.5 \times 10^{-11} \mathrm{erg} \mathrm{cm}^{-2} \mathrm{~s}^{-1}$ (in the $20-80 \mathrm{keV}$ energy range), so the quiescent emission is still likely to dominate the average spectrum.

To verify whether this is indeed the case, we also use data from the INGEGRAL IBIS (Lebrun et al. 2003) instrument including all public observations within $12^{\circ}$ from the source and excluding the outburst periods as determined from the BAT light curve (in particular, we exclude intervals when the Swift/BAT flux is greater than $5 \mathrm{mCrab}$ ). These results in a total of $1885 \mathrm{IN}-$ TEGRAL pointings and an effective exposure of $\sim 0.5 \mathrm{Ms}$. GK Per is not detected in individual pointings, so we extract the spectrum from the mosaic images obtained using all observations as recommended in the INTEGRAL data reduction guide ${ }^{5}$ for faint sources. We used the offline software analysis package OSA 10.1 and associated calibration files for the data reduction. The resulting spectrum has factor of two lower flux than the mission long Swift/BAT spectrum (i.e. $2.5 \times 10^{-11} \mathrm{erg} \mathrm{cm}^{-2} \mathrm{~s}^{-1}$ ), but otherwise there is no statistically significant difference in spectral shape. In particular, when fitted with optically thin bremsstrahlung models, the best-fit temperature is $20.1 \mathrm{keV}$ in both cases. Therefore we assume that both ISGRI and BAT spectra are representative of source properties in quiescence and simultaneously fit both with a free cross-normalization factor to account for the flux difference and instrumental discrepancies in absolute flux calibration.

\section{Results}

\subsection{EX Hya}

The broadband spectrum presented in Fig. 8 above $\sim 2 \mathrm{keV}$ is well described with the absorbed PSR model with the WD mass fixed to $0.79 M_{\odot}$ as found from binary motion (Beuermann \& Reinsch 2008). At lower energies there is a soft excess commonly observed in intermediate polars (Evans \& Hellier 2007), which can be accounted for either with a blackbody or APEC with $k T \sim 0.2 \mathrm{keV}$. Multiple narrow emission lines are known to be present in the soft band as well (Luna et al. 2010) and some are also apparent in the residuals of the XIS spectrum. To account for these, we added several Gaussians with zero widths and energies fixed to values reported by Luna et al. (2010) based on the high resolution Chandra spectra of the source. We also included a cross-normalization constant fixed to 1.18 for the PIN spectrum (as suggested in data reduction guide for PIN-nominal pointing). Other relevant parameters obtained from the fit are $R_{\mathrm{m}} / R=2.06_{-0.04}^{+0.05}$ and $N_{\mathrm{H}}=$ $4.1_{-0.7}^{+0.1} \times 10^{21} \mathrm{~cm}^{-2}$.

The hard X-ray continuum $(\gtrsim 3 \mathrm{keV})$ is adequately described by the PSR model. The spectra residuals below $\sim 2 \mathrm{keV}$ are caused by unmodelled line and continuum emission. We conclude, therefore, that the proposed model describes the X-ray continuum of the source well.

The X-ray continuum is mostly sensitive to WD parameters in the hard energy range, hence, the soft part of the spectrum does not actually help to eliminate the model degeneracy

http://www .isdc. unige.ch/integral/download/osa/doc 


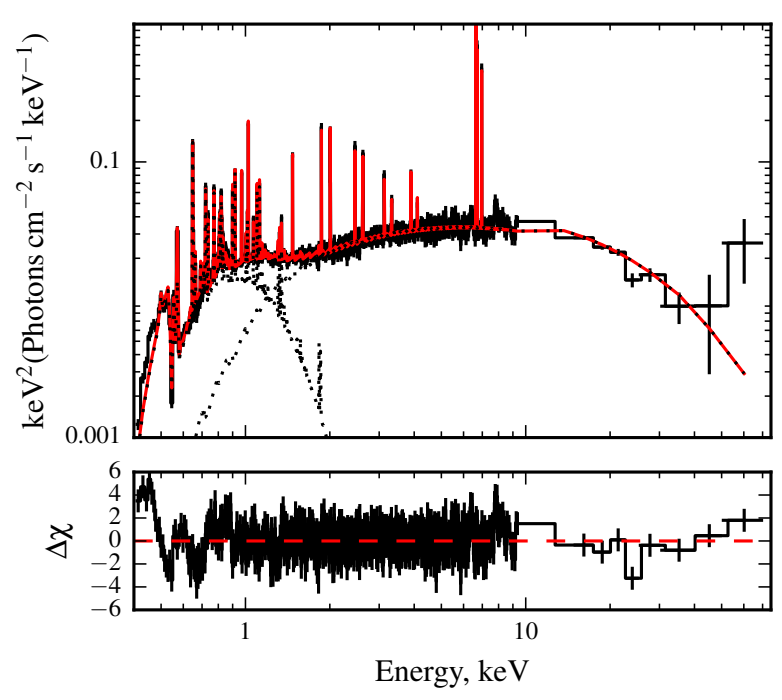

Fig. 8. Suzaku spectrum of EX Hya fitted with the PSR model spectrum with $M / M_{\odot}=0.79$ and $R_{\mathrm{m}} / R=2$.

between WD mass and magnetosphere radius. In fact, more detailed analysis similar to that by Luna et al. (2010) would be required to derive additional constraints on WD parameters from the soft X-ray spectra. Therefore, because of the strong absorption and lack of broadband quiescent observations, we only considered the spectrum above $20 \mathrm{keV}$ for GK Per (see below). Hereafter we ignore XIS data to estimate the mass of the WD in EX Hya. This approach allows us to verify whether the proposed method can provide adequate results using hard X-ray data alone.

We used the combined light curve with time resolution of $8 \mathrm{~s}$ from the three XIS units active during the observation to obtain the power spectrum of the source. As shown in Fig. 2, the power spectrum has a break at $v_{\mathrm{b}}=0.021 \pm 0.006 \mathrm{~Hz}$.

Next, we perform the two-parameter fitting of PIN data using model PSR spectra. The resulting strip, which corresponds to the best fit including formal errors, is shown in the $m-r_{\mathrm{m}}$ plane (see Fig. 9). The strip, which corresponds to the break frequency $v_{\mathrm{b}}$ with the uncertainties (see Fig. 2), is also shown. Crossing the two regions allows us to find the best-fit parameters of EX Hyd, $M / M_{\odot}=0.73 \pm 0.06$, and $R_{\mathrm{m}} / R=2.6 \pm 0.4$. The obtained parameters coincide within errors with the values obtained using other methods (see Sect. 4.1). Therefore, we conclude that the suggested method gives reliable results for EX Hya and can be used for other intermediate polars.

\subsection{GK Per}

\subsubsection{Outburst data}

Figure 10 shows the NuSTAR background subtracted light curves of the source in two energy bands. The source exhibits correlated variability in both energy bands on timescales of 5-10 ks, which resembles the quasi-periodical oscillations (QPOs) reported earlier (Watson et al. 1985; Ishida et al. 1996). However, we do not formally detect the QPOs directly in the power spectrum because of the data gaps in the NuSTAR observation that occur on the same timescale.

The most prominent features in the power spectrum of the 3-80 keV light curve are the two peaks, which are associated with the spin frequency and first harmonic, and the break at $v_{\mathrm{br}}=$ $0.0225 \pm 0.004$ (Fig 11).

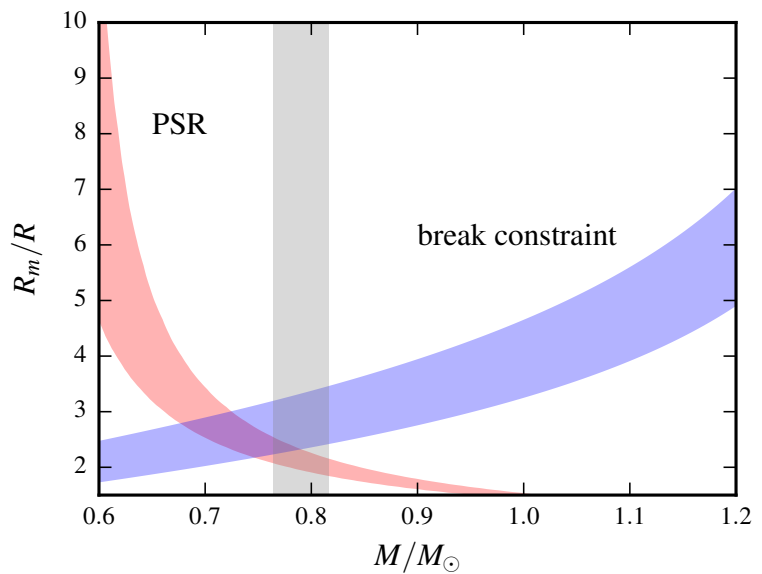

Fig. 9. Strips in the $m-r_{\mathrm{m}}$ plane obtained using spectral fitting and break frequency in the power spectrum of EX Hya. The vertical strip corresponds to the WD mass known from optical observations.

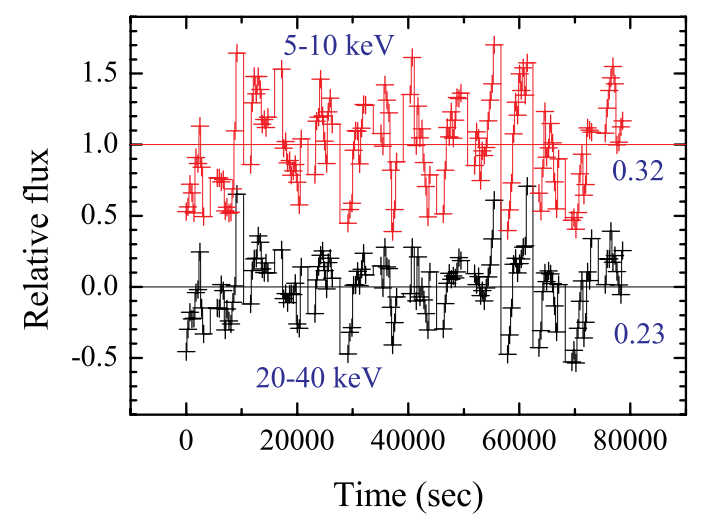

Fig. 10. Relative light curves of GK Per in two spectral bands, 5-10 keV and 20-40 keV, and time resolution of $351 \mathrm{~s}$. The fluxes in two bands are correlated, but the rms of the light curve in the soft band is higher (0.32) in comparison with the RMS of the light curve in the hard band (0.23).

Quasi-periodical oscillations were previously associated with obscuration of the emission from a white dwarf from bulges in the inner disc (Hellier \& Livio 1994). The amplitude of variability in the NuSTAR data decreases with increasing energy (see Fig. 10), which is also consistent with the hypothesis that the flux variability is largely driven by changes in the absorption column. To verify this hypothesis, we fitted the averaged spectrum presented in Fig. 12 with the PSR model modified by partial covering absorber (see e.g. discussion in Ramsay 2000). First, it is important to emphasize that the higher statistical quality of NuSTAR data makes it clear that this simple model is not really adequate to describe the broadband spectrum of the source below $20 \mathrm{keV}$. Nevertheless, the spectrum is described well by the model above $\sim 20 \mathrm{keV}$.

Also, the light curves folded with the spin period of the white dwarf show no significant dependence on the energy with the pulsed fraction $P F \approx 45 \%$ in all the energy bands. Therefore, the absorbing material is likely not located in the immediate vicinity of the WD. In addition, rapid variation of source hardness with time suggests that the absorption is variable on relatively short timescales and that the partial covering absorber is just a useful approximation to account for a constantly changing absorption column, which is likely caused by the obscuration of the emission region by outer parts of the accretion disc. 


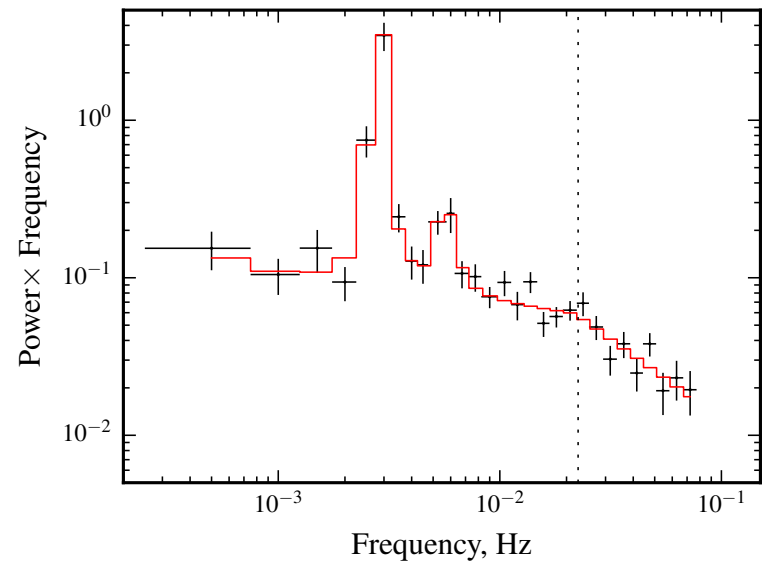

Fig. 11. Power spectrum of the X-ray light curve of GK Per observed by NuSTAR. The found break frequency $v_{\mathrm{b}}=0.0225 \pm 0.004$ is indicated with the vertical dotted line. The peaks are corresponded to the spin frequency and its first harmonic.

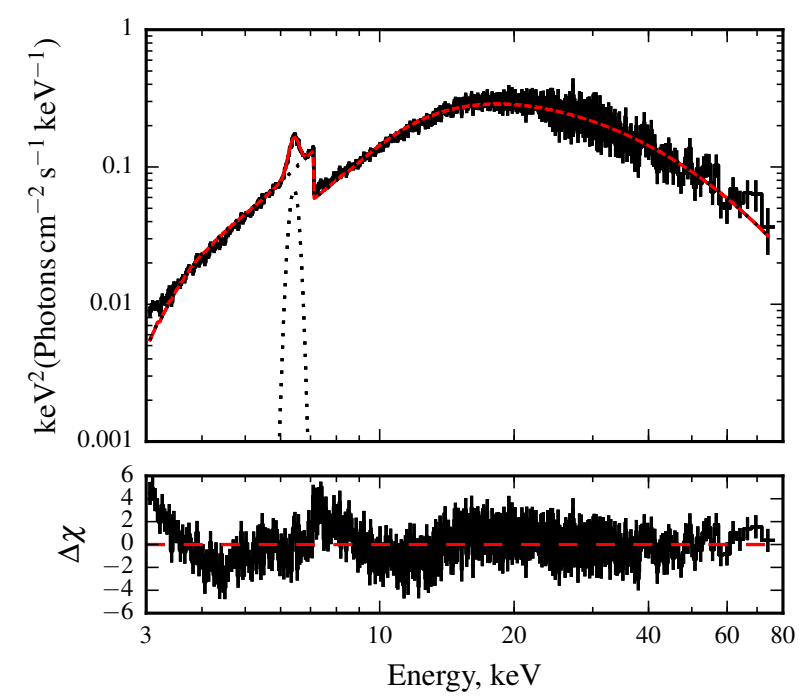

Fig. 12. Unfolded NuSTAR spectra of GK Per. The model spectrum was computed for fixed $M / M_{\odot}=0.86$. Other parameters found are $R_{\mathrm{m}} / R \approx 2.3$ and $N_{\mathrm{H}, 1} \approx 1.7 \times 10^{23} \mathrm{~cm}^{-2}$. The obtained partial covering parameters are $N_{\mathrm{H}, 2} \approx 1.24 \times 10^{24} \mathrm{~cm}^{-2}$ and covering fraction $C_{\mathrm{F}} \approx 0.82$ and $\chi_{\text {dof }}^{2} \approx 1.75$.

Moreover, the hard part of the spectrum is mostly sensitive to the WD parameters, whereas the largest fraction of photons is detected in the soft part that is dominated by complex absorption (as expected for the derived absorption column of $10^{23-24} \mathrm{~cm}^{-2}$ ). While it is possible to describe the broadband spectrum introducing several absorption columns, any ambiguity in modelling of the soft part of the spectrum is also likely to affect the hard part and thus the derived parameters of the WD simply because the soft part is much more important statistically. Therefore, to avoid any potential systematic effects associated with modelling of the soft part of the spectrum, we conservatively ignore data affected by the absorption below $20 \mathrm{keV}$ to determine the parameters of the WD.

The hard X-ray NuSTAR spectrum used to estimate the WD parameters in GK Per is shown in Fig. 13. The resulting strips in the $m-r_{\mathrm{m}}$ plane are shown in Fig. 14. The intersection region yields $M / M_{\odot}=0.86 \pm 0.02$ and $R_{\mathrm{m}} / R=2.8 \pm 0.2$. The value of the mass is consistent with the values determined

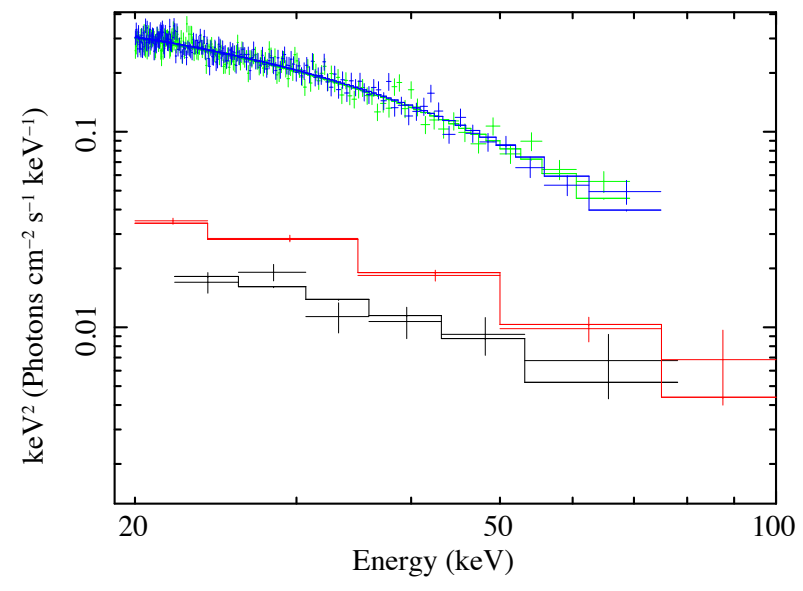

Fig. 13. Observed spectra of GK Per used for two-parameter spectral fitting, from top downwards: NuSTAR spectrum above $20 \mathrm{keV}$, Swift/BAT, and INTEGRAL spectra.

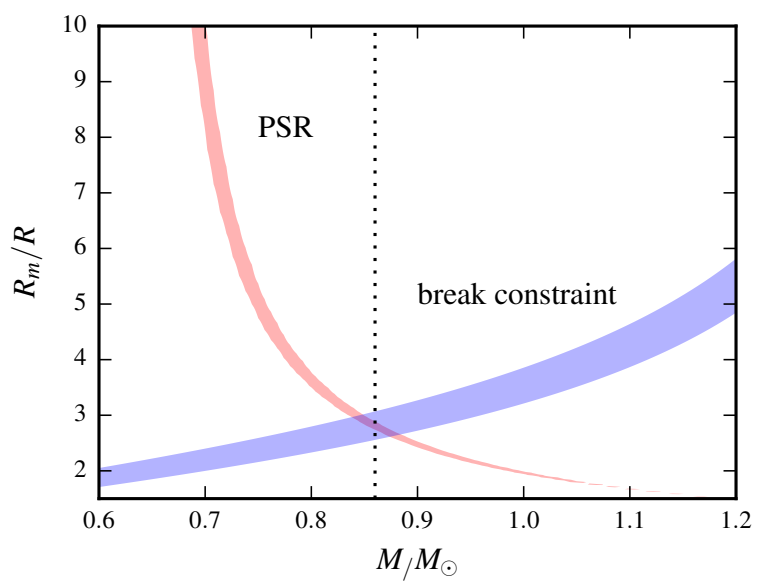

Fig. 14. Strips in the $m-r_{\mathrm{m}}$ plane obtained using spectral fitting NuSTAR spectrum and break frequency in the power spectrum of GK Per in outburst.

by other authors (see Sect. 4.2), but our measurement has much better accuracy.

\subsubsection{Magnetosphere size}

The hard X-ray luminosity of GK Per in quiescence is almost an order of magnitude lower in comparison with the outburst (Fig. 13). Fitting the spectra using the bremsstrahlung model yields $k T_{\mathrm{br}}=16.7 \pm 0.2 \mathrm{keV}$ (NuSTAR spectrum in the outburst), $k T_{\text {br }}=20.1 \pm 0.8 \mathrm{keV}$ (Swift/BAT averaged spectrum), and $k T_{\text {br }}=21_{-3}^{+4} \mathrm{keV}$ (INTEGRAL spectrum in the quiescence), i.e. when the luminosity decreases the magnetosphere size increases and, thus, the temperature increases. We fitted the Swift/BAT and INTEGRAL spectra simultaneously to quantify this effect and obtain the observed dependence $m-r_{\mathrm{m}}$ in quiescence. The result is shown in Fig. 15.

It is clear the magnetospheric radius in GK Per indeed increases in quiescence and equals $4.3_{-1}^{+2} R$ for $M=0.86 M_{\odot}$. Therefore, we can investigate how the magnetospheric radius depends on the observed X-ray flux and thus the accretion rate (Fig. 16). Here we used the observed flux of GK Per in the $20-80 \mathrm{keV}$ band $F_{20-80}$ as a tracer of the accretion rate. The magnetosphere size is expected to scale as some power 


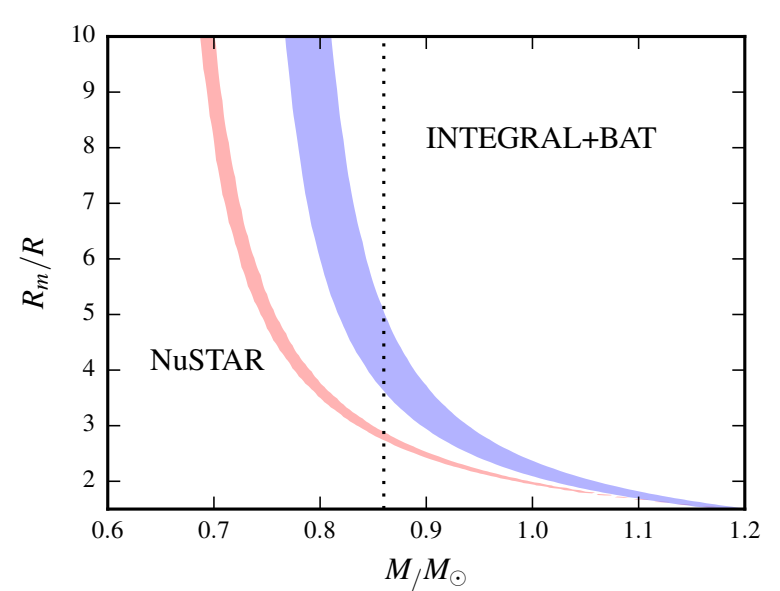

Fig. 15. Strips in the $m-r_{\mathrm{m}}$ plane obtained using spectral fitting of GK Per in outburst (NuSTAR spectrum) and quiescence (combined Swift/BAT and INTEGRAL spectrum).

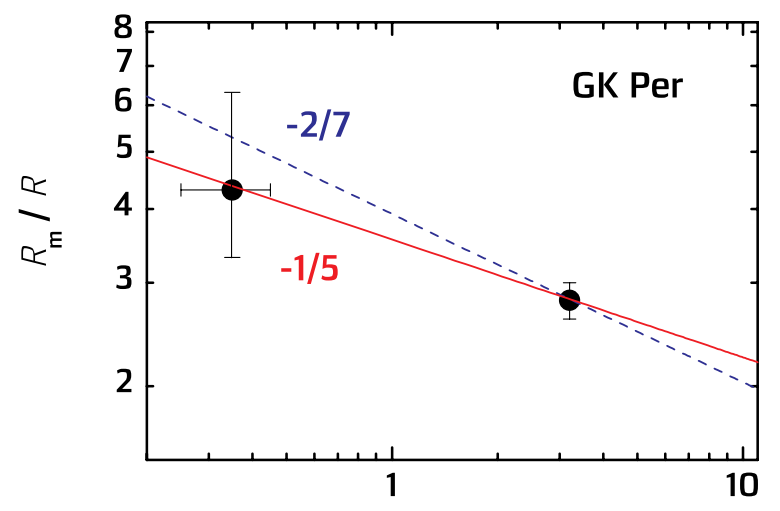

$$
\text { Observed flux } F_{20-80}\left(10^{-10} \mathrm{erg} \mathrm{s}^{-1} \mathrm{~cm}^{-2}\right)
$$

Fig. 16. Dependence of the GK Per magnetospheric radius on the observed flux in the $20-80 \mathrm{keV}$ band. The best fit with the slope $-1 / 5$ is shown together with the slope $-2 / 7$ inferred from the equation for the Alfv́en radius.

of luminosity or accretion rate. We evaluate the exponent $\beta$ in the $r_{\mathrm{m}} \sim\left(F_{20-80}\right)^{\beta}$ dependence to $-0.2_{-0.15}^{+0.10}$ using two obtained points on the $r_{\mathrm{m}}-F_{20-80}$ plane. Formally, the classical exponent in the equation for the Alfvén radius $\beta_{\mathrm{af}}=-2 / 7$ is fully consistent with the obtained value, which is rather uncertain as a result of low statistics in quiescence. In contrast, similar to our results, Kulkarni \& Romanova (2013) found a value that is somewhat lower than the classical value using 3D MHD simulations for small magnetospheres with $r_{\mathrm{m}}$ in the range $2.5-5$ as follows:

$\frac{R_{\mathrm{m}}}{R} \approx 1.06\left(\frac{\mu_{\mathrm{B}}^{4}}{\dot{M}^{2} G M R^{7}}\right)^{1 / 10}$,

where $\mu_{\mathrm{B}} \approx B R^{3}$ is the magnetic moment of the WD and $B$ is the magnetic field strength on its surface. Kulkarni \& Romanova (2013) explained this result with compressibility of the magnetosphere and remarked that this effect is less significant for larger magnetospheres. Currently our estimate is rather uncertain because of low statistics in quiescence and not constraining the theory. However, the conclusions by Kulkarni \& Romanova (2013) might become testable once better spectra of GK Per at low fluxes are available.

\section{Conclusions}

We suggested a new method for simultaneous determination of the white dwarf mass and the magnetospheric radius in intermediate polars. The method is based on two independent measurements of the degenerate $M-R_{\mathrm{m}}$ dependence using the observed break frequency in the power spectrum (see Revnivtsev et al. 2009, 2011) and the fitting of the hard X-ray spectrum with the newly developed PSR model, which takes into account the finite acceleration height of the accretion flow. The two measurements lead to two intersecting regions in the $M / M_{\odot}-R_{\mathrm{m}} / R$ plane that allow us to estimate the white dwarf mass and the relative magnetospheric radius.

For the spectral fitting procedure, we computed an extensive grid of two-parameter models for hard X-ray spectra of postshock regions on WD surfaces. We assumed quasi-dipole geometry of the PSR, fixed accretion rate $\left(\dot{M}=10^{16} \mathrm{~g} \mathrm{~s}^{-1}\right)$, and a polar region with fixed relative footprint $f=5 \times 10^{-4}$ of the WD surface. The cyclotron cooling and difference in temperatures of ion and electron plasmas are currently not taken into account. The WD mass range covered is $0.3-1.4 M_{\odot}$ (with steps of $\left.0.02 M_{\odot}\right)$. The second parameter of the model, the relative magnetospheric radius $r_{\mathrm{m}}=R_{\mathrm{m}} / R$, changes from 1.5 to 60 with steps proportional to $\left(1-r_{\mathrm{m}}^{-1}\right)$; we also included a model with $r_{\mathrm{m}}=1000$ to ease comparison with previously published results, i.e. the grid includes 41 values of $r_{\mathrm{m}}$. The model is implemented in the XSPEC package as an additive table model and is accessible to the scientific community.

The method was tested using the highly studied intermediate polar EX Hya. We obtained the WD mass of $(0.73 \pm 0.06) M_{\odot}$ and magnetospheric radius $R_{\mathrm{m}} / R=2.6 \pm 0.4$, which are fully consistent with the known WD mass and the magnetosphere size expected for this source. Subsequently we applied the method to another intermediate polar GK Per, which is also a dwarf nova. Large changes of flux during the outburst in GK Per allow us not only to estimate the WD mass and the relative magnetosphere size, but also to investigate the magnetosphere size changes with luminosity.

Using the NuSTAR observation of GK Per during an outburst at the flux level of $F_{20-80}=3.2 \times 10^{-10} \mathrm{erg} \mathrm{s}^{-1} \mathrm{~cm}^{-2}$ in the $20-80 \mathrm{keV}$ range, we estimate the WD mass to $M / M_{\odot}=$ $0.86 \pm 0.02$ and $R_{\mathrm{m}} / R=2.8 \pm 0.2$. The fit to the combined Swift/BAT and INTEGRAL spectra of GK Per in quiescence gives $R_{\mathrm{m}} / R=4.3_{-1}^{+2}$ at fixed $M$ and $F_{20-80}=3.5 \times 10^{-11} \mathrm{erg}$ $\mathrm{s}^{-1} \mathrm{~cm}^{-2}$. The derived $R_{\mathrm{m}} / R-\left(F_{20-80}\right)^{\beta}$ dependence with $\beta=$ $-0.2_{-0.15}^{+0.10}$ is consistent with the classical dependence for Alfvén radius and with the results obtained by Kulkarni \& Romanova (2013) for small magnetospheres from MHD simulations. We note that it could be possible to test the predictions by these authors once quiescent hard spectra of GK Per with better statistical quality are available.

Acknowledgements. This work made use of data from the NuSTAR mission, a project led by the California Institute of Technology, managed by the Jet Propulsion Laboratory, and funded by the National Aeronautics and Space Administration. This paper is also based on data from observations with INTEGRAL, an ESA project with instruments and science data centre funded by ESA member states (especially the PI countries: Denmark, France, Germany, Italy, Spain, and Switzerland), Czech Republic and Poland, and with the participation of Russia and the USA. This research has made use of data obtained from the Suzaku satellite, a collaborative mission between the space agencies of Japan (JAXA) and the USA (NASA). V.S. thanks Deutsche Forschungsgemeinschaft (DFG) for financial support (grant WE 1312/48-1). V.D. and L.D. acknowledges support by the Bundesministerium für Wirtschaft und Technologie and the Deutsches Zentrum für Luft und Raumfahrt through the grant FKZ 50 OG 1602. 


\section{References}

Aizu, K. 1973, Prog. Theor. Phys., 49, 1184

Barlow, E. J., Knigge, C., Bird, A. J., et al. 2006, MNRAS, 372, 224

Barthelmy, S. D., Barbier, L. M., Cummings, J. R., et al. 2005, Space Sci. Rev., 120,143

Baumgartner, W. H., Tueller, J., Markwardt, C. B., et al. 2013, ApJS, 207, 19

Belle, K. E., Howell, S. B., Sion, E. M., Long, K. S., \& Szkody, P. 2003, ApJ, 587,373

Beuermann, K., \& Reinsch, K. 2008, A\&A, 480, 199

Beuermann, K., Harrison, T. E., McArthur, B. E., Benedict, G. F., \& Gänsicke, B. T. 2003, A\&A, 412, 821

Brunschweiger, J., Greiner, J., Ajello, M., \& Osborne, J. 2009, A\&A, 496, 121

Canalle, J. B. G., Saxton, C. J., Wu, K., Cropper, M., \& Ramsay, G. 2005, A\&A 440,185

Crampton, D., Fisher, W. A., \& Cowley, A. P. 1986, ApJ, 300, 788

Cropper, M., Ramsay, G., \& Wu, K. 1998, MNRAS, 293, 222

Cropper, M., Wu, K., Ramsay, G., \& Kocabiyik, A. 1999, MNRAS, 306, 684

Evans, P. A., \& Hellier, C. 2007, ApJ, 663, 1277

Ezuka, H., \& Ishida, M. 1999, ApJS, 120, 277

Fabian, A. C., Pringle, J. E., \& Rees, M. J. 1976, MNRAS, 175, 43

Falanga, M., Bonnet-Bidaud, J. M., \& Suleimanov, V. 2005, A\&A, 444, 561

Hachisu, I., \& Kato, M. 2007, ApJ, 662, 552

Hayashi, T., \& Ishida, M. 2014a, MNRAS, 438, 2267

Hayashi, T., \& Ishida, M. 2014b, MNRAS, 441, 3718

Hellier, C., \& Livio, M. 1994, ApJ, 424, L57

Hellier, C., \& Sproats, L. N. 1992, IBVS, 3724

Hellier, C., Harmer, S., \& Beardmore, A. P. 2004, MNRAS, 349, 710

Ibragimov, A. A., Suleimanov, V. F., Vikhlinin, A., \& Sakhibullin, N. A. 2003, Astron. Rep., 47, 186

Ishida, M., Sakao, T., Makishima, K., et al. 1992, MNRAS, 254, 647

Ishida, M., Yamashita, A., Ozawa, H., Nagase, F., \& Inoue, H. 1996, IAU Circ. 6340

Kokubun, M., Makishima, K., Takahashi, T., et al. 2007, PASJ, 59, 53

Koyama, K., Tsunemi, H., Dotani, T., et al. 2007, PASJ, 59, 23

Kulkarni, A. K., \& Romanova, M. M. 2013, MNRAS, 433, 3048

Kurucz, R. L. 1970, SAO Special Report, 309

Kurucz, R. 1993, ATLAS9 Stellar Atmosphere Programs and $2 \mathrm{~km} \mathrm{~s}^{-1}$ grid, Kurucz CD-ROM No. 13 (Cambridge, Mass.: Smithsonian Astrophysical Observatory)

Landi, R., Bassani, L., Dean, A. J., et al. 2009, MNRAS, 392, 630

Lebrun, F., Leray, J. P., Lavocat, P., et al. 2003, A\&A, 411, L141

Luna, G. J. M., Raymond, J. C., Brickhouse, N. S., et al. 2010, ApJ, 711, 1333

Luna, G. J. M., Raymond, J. C., Brickhouse, N. S., Mauche, C. W., \& Suleimanov, V. 2015, A\&A, 578, A15

Mauche, C. W. 2004, in Magnetic Cataclysmic Variables, IAU Colloq. 190: eds. S. Vrielmann, \& M. Cropper, ASP Conf. Ser., 315, 120
McLaughlin, D. B. 1960, in Stellar Atmospheres, ed. J. L. Greenstein, 585 Mihalas, D. 1978, Stellar atmospheres, 2nd edn. (San Francisco: Freeman) Morales-Rueda, L., Still, M. D., \& Roche, P. 1999, MNRAS, 306, 753 Morales-Rueda, L., Still, M. D., Roche, P., Wood, J. H., \& Lockley, J. J. 2002, MNRAS, 329, 597

Nauenberg, M. 1972, ApJ, 175, 417

Neustroev, V. V., Suleimanov, V. F., Borisov, N. V., Belyakov, K. V., \& Shearer, A. 2011, MNRAS, 410, 963

Norton, A. J., Watson, M. G., \& King, A. R. 1988, MNRAS, 231, 783

Patterson, J. 1991, PASP, 103, 1149

Patterson, J., Kemp, J., Richman, H. R., et al. 1998, PASP, 110, 415

Ramsay, G. 2000, MNRAS, 314, 403

Reinsch, K. 1994, A\&A, 281, 108

Revnivtsev, M., Lutovinov, A., Suleimanov, V., Sunyaev, R., \& Zheleznyakov, V. 2004a, A\&A, 426, 253

Revnivtsev, M. G., Lutovinov, A. A., Suleimanov, B. F., Molkov, S. V., \& Sunyaev, R. A. 2004b, Astron. Lett., 30, 772

Revnivtsev, M., Churazov, E., Postnov, K., \& Tsygankov, S. 2009, A\&A, 507, 1211

Revnivtsev, M., Potter, S., Kniazev, A., et al. 2011, MNRAS, 411, 1317

Rothschild, R. E., Gruber, D. E., Knight, F. K., et al. 1981, ApJ, 250, 723

Saxton, C. J., Wu, K., Canalle, J. B. G., Cropper, M., \& Ramsay, G. 2007, MNRAS, 379, 779

Siegel, N., Reinsch, K., Beuermann, K., Wolff, E., \& van der Woerd, H. 1989, A\&A, 225, 97

Smith, R. K., Brickhouse, N. S., Liedahl, D. A., \& Raymond, J. C. 2001, ApJ, 556, L91

Suleimanov, V., \& Werner, K. 2007, A\&A, 466, 661

Suleimanov, V., Revnivtsev, M., \& Ritter, H. 2005, A\&A, 435, 191

Suleimanov, V., Poutanen, J., Falanga, M., \& Werner, K. 2008, A\&A, 491, 525

Sutherland, R. S., \& Dopita, M. A. 1993, ApJS, 88, 253

Takahashi, T., Abe, K., Endo, M., et al. 2007, PASJ, 59, 35

Simon, V. 2002, A\&A, 382, 910

Vrielmann, S., Ness, J.-U., \& Schmitt, J. H. M. M. 2005, A\&A, 439, 287

Warner, B. 1976, in Structure and Evolution of Close Binary Systems, eds. P. Eggleton, S. Mitton, \& J. Whelan, IAU Symp., 73, 85

Warner, B. 1986, MNRAS, 222, 11

Warner, B. 2003, Cataclysmic Variable Stars (Cambridge University Press)

Watson, M. G., King, A. R., \& Osborne, J. 1985, MNRAS, 212, 917

Winkler, C., Courvoisier, T. J.-L., Di Cocco, G., et al. 2003, A\&A, 411, L1

Wu, K., Chanmugam, G., \& Shaviv, G. 1994, ApJ, 426, 664

Yuasa, T., Nakazawa, K., Makishima, K., et al. 2010, A\&A, 520, A25

Yuasa, T., Hayashi, T., \& Ishida, M. 2016, MNRAS, 459, 779

Zemko, P., Orio, M., \& Evans, P. A. 2015, ATel, 7404

Zemko, P., Orio, M., Luna, G. J. M., \& Mukai, K. 2016, ArXiv e-prints [arXiv: 1603.03286] 


\section{Appendix A: Compare with the previous paper}

As mentioned by Hayashi \& Ishida (2014a), in Suleimanov et al. (2005) the total number density $n$ was used instead of both the ion number density $n_{\mathrm{i}}$ and the electron number density $n_{\mathrm{e}}$. That replacement increased the cooling rate in the considered accretion column models by approximately four times, generating a column model four times shorter (see Fig. A.1, bottom panel). This led to a different emergent spectrum normalization (four times smaller). Fortunately, the shapes of the computed spectra were not affected (see Fig. A.1, top panel). Consequently, the WD masses derived in the Suleimanov et al. (2005) remain correct because they are determined by the shape of the spectrum alone. We note that this error was found just after the publication and the spectrum grid used by Brunschweiger et al. (2009) was recomputed with a corrected version of the code. Models computed with Compton scattering taken into consideration (Suleimanov et al. 2008) were also computed using the correct cooling rate.
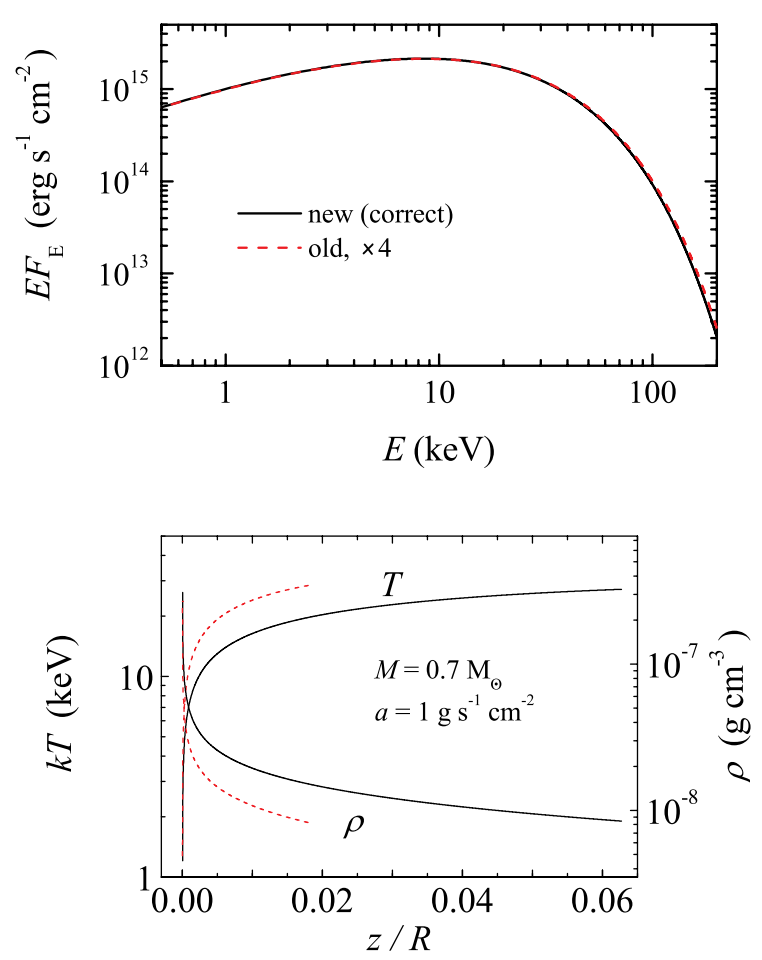

Fig. A.1. Old and new spectra (top panel) as well as temperature and density stratifications (bottom panel). 